\title{
Transplantation versus Resection for Hilar Cholangiocarcinoma: An Argument for Shifting Treatment Paradigms for Resectable Disease
}

Cecilia G. Ethun, MD¹, Alexandra G. Lopez-Aguiar, MD¹, Douglas J. Anderson, MD², Andrew B. Adams, MD, $\mathrm{PhD}^{2}$, Ryan C. Fields, $\mathrm{MD}^{3}$, Maria B. Doyle, $\mathrm{MD}^{4}$, William C. Chapman, MD $^{4}$, Bradley A. Krasnick, MD ${ }^{3}$, Sharon M. Weber, MD $^{5}$, Joshua D. Mezrich, MD $^{6}$, Ahmed Salem, MD ${ }^{5}$, Timothy M. Pawlik, MD, MPH, PhD ${ }^{7}$, George Poultsides, MD $^{8}$, Thuy B. Tran, MD $^{8}$, Kamran Idrees, MD $^{9}$, Chelsea A. Isom, MD $^{9}$, Robert C.G. Martin, MD ${ }^{10}$, Charles R. Scoggins, $\mathbf{M D}^{10}$, Perry Shen, $\mathbf{M D}^{11}$, Harveshp D. Mogal, MD ${ }^{11}$, Carl Schmidt, MD ${ }^{12}$, Eliza Beal, MD ${ }^{12}$, loannis Hatzaras, MD ${ }^{13}$, Rivfka Shenoy, MD $^{13}$, Kenneth Cardona, MD ${ }^{1}$, and Shishir K. Maithel, MD ${ }^{1}$

${ }^{1}$ Division of Surgical Oncology, Department of Surgery, Winship Cancer Institute, Emory University, Atlanta, GA

${ }^{2}$ Division of Transplantation, Department of Surgery, Emory University, Atlanta GA

${ }^{3}$ Department of Surgery, Section of Hepatobiliary, Pancreatic and Gastrointestinal Surgery, Washington University School of Medicine, St Louis, MO

${ }^{4}$ Department of Surgery, Section of Abdominal Transplantation, Washington University School of Medicine, St Louis, MO

${ }^{5}$ Division of Surgical Oncology, Department of Surgery, University of Wisconsin School of Medicine and Public Health, Madison, WI

${ }^{6}$ Division of Transplant, Department of Surgery, University of Wisconsin School of Medicine and Public Health, Madison, WI

${ }^{7}$ Division of Surgical Oncology, Department of Surgery, The Johns Hopkins Hospital, Baltimore, MD

${ }^{8}$ Department of Surgery, Stanford University Medical Center, Stanford, CA

${ }^{9}$ Division of Surgical Oncology, Department of Surgery, Vanderbilt University Medical Center, Nashville, TN

${ }^{10}$ Division of Surgical Oncology, Department of Surgery, University of Louisville, Louisville, KY

${ }^{11}$ Department of Surgery, Wake Forest University, Winston-Salem, NC

${ }^{12}$ Division of Surgical Oncology, The Ohio State University, Columbus, $\mathrm{OH}$

${ }^{13}$ Department of Surgery, New York University, New York, NY

Corresponding Author / Contact For Reprints: Shishir K. Maithel, MD, FACS, 1365 C Clifton Road NE, $2^{\text {nd }}$ Floor, Atlanta, Georgia 30322, Tel: 404.778.5777| Fax: 404.778.4225, smaithe@emory.edu. 


\section{Abstract}

Objective-To investigate the influence of type of surgery (transplant versus resection) on overall survival (OS) in patients with hilar cholangiocarcinoma (H-CCA).

Background-Outcomes after resection for H-CCA are poor, yet transplantation is currently only reserved for well-selected patients with unresectable disease.

Methods-All patients with H-CCA who underwent resection from 2000-2015 at 10-institutions were included. Three institutions additionally had active H-CCA transplant protocols with similar selection criteria over similar time-periods.

Results-Of 304pts with suspected H-CCA, 234 underwent attempted resection and 70 were enrolled in a transplant protocol. Excluding incomplete/R2 resections $(n=43)$, patients who were enrolled but did not undergo transplant $(\mathrm{n}=24)$, and transplants without confirmed H-CCA diagnoses ( $n=5), 191$ pts underwent curative-intent resection and 41 curative-intent transplant. Compared to resection, transplant patients were younger (52vs65yrs; $\mathrm{p}<0.001)$, and more frequently had primary sclerosing cholangitis (PSC: 61vs2\%; $<<0.001)$ and received chemotherapy and/or radiation (98vs57\%; $\mathrm{p}<0.001)$. Groups were otherwise similar in demographics and comorbidities. Patients who underwent transplant for confirmed H-CCA diagnosis had improved OS compared to resection (3-yr: 72vs33\%; 5-yr: 64vs18\%; p<0.001). Among patients who underwent resection for tumors $<3 \mathrm{~cm}$ with lymph-node negative disease, and excluding PSC patients, transplant was still associated with improved OS (3-yr: 54vs44\%; 5-yr: $54 \mathrm{vs} 29 \% ; \mathrm{p}=0.03)$. Transplant remained associated with improved survival on intention-to-treat analysis, even after accounting for tumor size, LN status, and PSC ( $\mathrm{p}=0.049)$.

Conclusions-Resection for hilar cholangiocarcinoma that meets criteria for transplantation $(<3 \mathrm{~cm}$, lymph-node negative disease) is associated with substantially decreased survival compared to transplant for the same criteria with unresectable disease. Prospective trials are needed and justified.

\section{Introduction}

Hilar cholangiocarcinoma (H-CCA) is a rare disease. With approximately 5,000 new cases diagnosed annually in the United States, H-CCA represents less than $2 \%$ of all malignancies, yet accounts for over $60 \%$ of all cholangiocarcinomas. ${ }^{1,2}$ Primary sclerosing cholangitis (PSC) is the most well-established risk factor, with nearly one-third of patients with PSC developing cholangiocarcinoma, the majority of whom develop it within the first few years of their PSC diagnosis. ${ }^{3}$ Still, most patients present with de novo H-CCA, without any identifiable underlying cause. ${ }^{4}$

Surgery is the only potentially-curative treatment option for H-CCA and, in the absence of metastatic or locally-advanced disease, or PSC, resection is considered the standard-of-care, with the primary goal of an $\mathrm{R} 0$ resection. ${ }^{2}$ Yet, despite advances in surgical technique, resection for H-CCA can be challenging. Negative margins are only achieved $60-80 \%$ of the time, and post-operative morbidity rates have been reported as high as $70 \% .^{5-9} \mathrm{In}$ addition, over half of patients have disease recurrence after curative-intent resection, and long-term survival remains poor, ranging from $20 \%$ to $40 \%$ at 5 years. ${ }^{7-13}$ Among patients who 
undergo R1 resections, 5-year survival approaches zero. ${ }^{13-15}$ Perhaps most sobering, however, is that only $40 \%$ of patients who present with H-CCA even undergo resectionapproximately $20 \%$ of patients have unresectable disease at the time of presentation, and of the $80 \%$ who undergo operative exploration, only half are ultimately deemed resectable. ${ }^{9,13}$ Although marginal improvements in survival have been seen with palliative chemotherapy, most patients with H-CCA who do not undergo curative-intent surgery unfortunately succumb to the disease within one year. ${ }^{13,16}$

Orthotopic liver transplantation was proposed in the late 1980s as a therapeutic option that obviated the concerns with achieving negative surgical margins, and could be offered to patients who were not candidates for operative resection due to locally-advanced or unresectable disease. Despite dismal initial results, pioneering work from the Mayo Clinic established a successful multimodality protocol for unresectable H-CCA, based on a strategy of neoadjuvant therapy with high-dose radiation and chemotherapy, followed by operative staging, and finally, liver transplantation. ${ }^{17-20}$ Among highly selected patients, including those with unresectable, solitary tumors, less than $3 \mathrm{~cm}$ in radial diameter, without evidence of lymph node metastases, and those with resectable disease in the setting of PSC, investigators from the Mayo Clinic reported a 5-year survival of $82 \%$ after transplantation. ${ }^{20}$ The Mayo protocol has since been adopted by over a dozen transplant programs, and similarly favorable results have been demonstrated. ${ }^{21}$

Despite the encouraging results in patients with unresectable disease, the role of neoadjuvant therapy followed by liver transplantation for resectable H-CCA is controversial. Concerns regarding organ allocation, wait times, and the intensity of the neoadjuvant protocol have been significant barriers to adopting this practice. Furthermore, whether there would be a significant survival benefit with liver transplantation over resection for resectable disease is unclear, as few centers outside the Mayo Clinic have the volume and experience with liver transplantation for H-CCA necessary to examine outcomes. Thus, our aim was to utilize a large, multi-institutional database to compare clinicopathologic factors and survival between patients who underwent resection and those who underwent liver transplantation for H-CCA.

\section{Methods}

The data for patients undergoing resection was obtained from a retrospective database from the U.S. Extrahepatic Biliary Malignancy Consortium (USEBMC), which is a collaboration of 10 academic institutions: Emory University, Johns Hopkins University, New York University, The Ohio State University, Stanford University, University of Louisville, University of Wisconsin, Vanderbilt University, Wake Forest University, and Washington University in St. Louis. All patients with H-CCA who underwent exploration and/or resection from January 2000 to March 2015 were evaluated.

Three of the 10 institutions additionally had active H-CCA transplant protocols during the same time-period: Emory University (initiated in 2009), University of Wisconsin (initiated in 2005), and Washington University in St. Louis (initiated in 2006). All patients from these institutions who entered an H-CCA transplant protocol from January 2005 to August 2016 were evaluated. The H-CCA transplant protocols at these institutions were nearly identical 
and all were based on the previously published Mayo protocol, including strict diagnostic, inclusion, and exclusion criteria, neoadjuvant chemoradiation (+/- brachytherapy), and either operative staging prior to or at the time of transplantation. ${ }^{19,20}$ Specifically, all protocols excluded patients with tumors greater than $3 \mathrm{~cm}$ in radial diameter on preoperative imaging and those with evidence of lymph node metastases. All patients who were listed for transplant received Model of End-stage Liver Disease (MELD) exception points, based on the 2002 United Network for Organ Sharing / Organ Procurement and Transplantation Network (UNOS/OPTN) policy for transplant allocation. ${ }^{22}$

Pertinent baseline demographic, peri-operative, pathologic, and survival data were collected. Confirmation of diagnosis was determined either on pre-operative pathologic/cytologic analysis or from pathologic analysis of the resected/explanted specimen. Patients with clinical, but no pathologic, diagnoses of H-CCA were deemed to have unconfirmed diagnoses. Pathologic review was performed by experienced GI pathologists at the time of surgery at each institution. Pathologic staging was defined as per American Joint Committee on Cancer (AJCC) 7th edition guidelines. ${ }^{23}$ The Social Security Death Index was used to verify survival information, when necessary. Institutional Review Board approval was obtained at each institution prior to data collection.

The primary objective was to assess the association between type of surgery (neoadjuvant therapy/transplant versus resection) and overall survival (OS), which was defined as the time from surgery to death from any cause or last known follow-up. As a secondary endpoint, intention-to-treat OS was calculated from either the date of surgery (resection) or the date of protocol initiation (transplant) to death from any cause or last known follow-up.

All statistical analysis was conducted using SPSS 23.0 software (IBM Inc., Armonk, NY). Patients undergoing neoadjuvant therapy/transplantation and resection were compared using chi-squared analyses and Mann-Whitney U tests, where appropriate. Log-rank analyses were performed to compare OS between transplant and resection cohorts. Statistical significance was defined as two-tailed $\mathrm{p}<0.05$ for all endpoints.

\section{Results}

Of 304 patients with H-CCA, 234 (77\%) underwent attempted resection and, excluding aborted and R2 resections ( $\mathrm{n}=43), 191(62 \%)$ underwent curative-intent resection (Figure 1). Seventy patients (23\%) were enrolled in a transplant protocol. Of those, $61(87 \%)$ completed the protocol (neoadjuvant chemoradiation) and were listed for transplant. Twelve patients (17\%) who completed neoadjuvant therapy and were initially listed, were removed from the transplant list while waiting. Forty-nine (70\%) underwent attempted transplantation and 46 $(66 \%)$ were successful. Finally, 5 patients $(7 \%)$ did not have confirmed diagnoses of H-CCA and were excluded, leaving 41 patients (59\%) who underwent transplantation for pathologically-confirmed H-CCA for final analysis (Figure 1).

Comparative analyses of baseline demographics and clinicopathologic factors between patients who underwent curative-intent resection $(n=191)$ and those who underwent successful transplantation $(\mathrm{n}=41)$ are shown in Table 1. Compared to resection, patients who 
underwent transplantation were younger (54 vs 67 years; $\mathrm{p}<0.001$ ), and more likely to have concurrent PSC (61\% vs $2 \%$; $\mathrm{p}<0.001)$. Transplantation was also associated with a lower incidence of any postoperative complication compared to resection ( $49 \%$ vs $68 \%$; $\mathrm{p}=0.03$ ), but there was no difference between groups in major complications ( $34 \%$ vs $45 \%$; $\mathrm{p}=0.30$ ), postoperative liver failure ( $10 \%$ vs $7 \%$; $\mathrm{p}=0.50)$, 90 -day mortality ( $5 \%$ vs $12 \% ; \mathrm{p}=0.27$ ).

Twelve patients (29\%) who underwent transplant had pathologic complete response on explant. Compared to resection, transplantation was associated with less frequent R1 resections ( $10 \%$ vs $30 \%$; $\mathrm{p}=0.01)$, and lymphovascular ( $18 \%$ vs $41 \%$; $\mathrm{p}=0.02)$ and perineural invasion ( $33 \%$ vs $78 \%$; $<0.001$ ). Despite similar lymph node retrieval, patients who underwent transplant were less likely to have lymph node positive disease compared to those who underwent resection ( $19 \%$ vs $38 \%$; $\mathrm{p}=0.04$ ). Neoadjuvant therapy was more common in patients who underwent transplant, while adjuvant therapy was more common among patients who underwent resection (all $\mathrm{p}<0.001$ ). There was no difference between transplant and resection groups in the incidence of disease recurrence ( $24 \%$ vs $37 \% ; \mathrm{p}=0.19)$, which was most commonly distant disease recurrence in both groups ( $80 \%$ vs $71 \% ; \mathrm{p}=0.72$ ). However, transplant patients had fewer isolated locoregional combined with liver only recurrences compared to resection patients ( $20 \%$ vs $62 \%$; $\mathrm{p}=0.02$; Table 1 ).

Comparing all transplant patients with pathologically-confirmed H-CCA $(n=41)$ to those who underwent curative-intent resection $(\mathrm{n}=191)$, transplantation was associated with improved OS (MNR vs 21.0 months, 95\% CI 16.5-25.5; p<0.001; Figure 2a). Median follow-up for survivors in the entire cohort was 23 months (range, 0.3-127); 58 months (range, 3-127) for patients who underwent transplant, and 15 months (range, 0.3-98) for patients who underwent resection.

Comparing these same transplant patients with pathologically-confirmed H-CCA to those who underwent curative-intent resection but had tumors $<3 \mathrm{~cm}$ in size and no lymph node disease (N0) on pathologic examination ( $\mathrm{n}=57$ ), still transplantation was associated with improved OS (MNR vs 27.4 months, 95\% CI 6.0-48.8; p<0.001; Figure 2b).

Further subset analysis was performed to compare $<3 \mathrm{~cm} / \mathrm{N} 0$ resection patients to those who underwent neoadjuvant therapy/transplant, excluding all patients with a diagnosis of PSC (resection $\mathrm{n}=1$; transplant $\mathrm{n}=25$ ), shown in Table 2. Non-PSC transplant patients were younger (60 vs 67 years; $\mathrm{p}=0.01$ ), less likely to have $\mathrm{R} 1$ resections ( $19 \%$ vs $30 \%$; $\mathrm{p}=0.01$ ) and tumors with perineural invasion (50\% vs $81 \%$; $\mathrm{p}=0.04)$, but trended toward a larger median tumor size compared to $<3 \mathrm{~cm} / \mathrm{N} 0$ resection patients ( $2.9 \mathrm{vs} 2.0 \mathrm{~cm} ; \mathrm{p}=0.08$ ). None of the non-PSC transplant patients had pathologic complete response, and 19\% had lymph node positive disease. The incidence of disease recurrence was also similar between nonPSC transplant and $<3 \mathrm{~cm} / \mathrm{N} 0$ resection patients ( $31 \%$ vs $29 \%$; $\mathrm{p}=1.00$ ).

Curative-intent transplantation for pathologically-confirmed H-CCA in non-PSC patients was associated with improved median OS compared to curative-intent $<3 \mathrm{~cm} / \mathrm{N} 0$ resection patients (MNR vs 25.9 months, 95\% CI 13.0-38.7; p=0.03; Figure 3a). When examining only $<3 \mathrm{~cm} / \mathrm{N} 0$ resection patients who also had $\mathrm{R} 0$ resections $(\mathrm{n}=39)$, still, non-PSC 
transplantation was associated with improved OS (MNR vs 28.3 months, 95\% CI 12.6-44.0; $\mathrm{p}=0.049$; Figure $3 \mathrm{~b})$.

An intention-to-treat analysis was performed on all patients who underwent attempted resection $(\mathrm{n}=234)$ and those who were enrolled in a transplant protocol for suspected $\mathrm{H}$ CCA, with or without pathologic confirmation $(n=66)$. Four patients who were enrolled in a transplant protocol but did not receive a transplant were missing protocol initiation dates, and were excluded from survival analysis (three patients with PSC-associated disease; one patient with de novo disease, who died of other causes without evidence of disease progression). Transplantation was associated with improved OS (median 77.4 months, 95\% CI not reported) compared to resection (median 17.1 months, 95\% CI 17.8-26.3; p<0.001). One-, 3-, and 5-year OS for transplant and resection patients were 80\%, 58\%, and 53\%, respectively, and 66\%,29\%, and 17\%, respectively. Transplantation was still associated with improved OS, even when comparing all attempted resections for $<3 \mathrm{~cm} / \mathrm{N} 0$ patients with all patients enrolled in a transplant protocol ( $\mathrm{p}=0.002$; Figure $4 \mathrm{a})$, as well as with all non-PSC patients enrolled in a transplant protocol ( $\mathrm{p}=0.049$; Figure $4 \mathrm{~b})$.

\section{Discussion}

Hilar cholangiocarcinoma is a rare disease with a poor prognosis, and surgery is the only potentially-curative treatment option. For patients with resectable disease, surgical resection is the current standard-of-care. Neoadjuvant therapy followed by transplantation is reserved for a highly select group of patients (including those with tumors $<3 \mathrm{~cm}$ in size and no lymph node involvement) with unresectable disease and patients with underlying PSC. ${ }^{2}$ The role of neoadjuvant therapy/liver transplantation in patients with resectable disease is not known. In this study, we utilized a large, 10-institution database of patients with H-CCA who underwent resection, as well as those who were enrolled in a transplant protocol, to investigate the influence of management strategy (neoadjuvant therapy/transplant versus resection) on OS. We found that, among patients undergoing curative-intent surgery, neoadjuvant therapy/transplantation was associated with improved OS, even when compared to resection for tumors $<3 \mathrm{~cm}$ and N0 disease, and with negative margins, as well as when excluding patients with a PSC diagnosis. On intention-to-treat analysis, neoadjuvant therapy/ transplantation was still associated with improved OS, even when compared to resection for tumors $<3 \mathrm{~cm}$ and $\mathrm{N} 0$ disease, and when excluding patients with PSC.

The surgical management of $\mathrm{H}-\mathrm{CCA}$ is challenging, and outcomes following resection have been suboptimal. In a study by Matsuo et al. of 380 patients with H-CCA who were evaluated and treated at Memorial Sloan Kettering Cancer Center from 1991 to 2008, 22\% of patients were unresectable at presentation, and of those who were explored, only 53\% ultimately underwent resection. Even with curative-intent resection, only $76.4 \%$ of patients achieved negative margins, $59.2 \%$ had postoperative complications, and $7.6 \%$ died in the perioperative period. ${ }^{13}$ Other studies have shown similar results. ${ }^{5-9}$ Beyond the technical challenges of resection, the oncologic outcomes for H-CCA are poor. ${ }^{12-15}$ Groot Koerkamp and colleagues estimated a $76 \%$ disease recurrence rate at 8 years following resection, and reported a 14\% 10-year recurrence-free survival in their study of 306 patients with H-CCA.

${ }^{12}$ Survival following resection falls in the $20-40 \%$ range at 5 years in most large series, and 
has remained constant over several decades, despite improved operative techniques and perioperative care. ${ }^{5,8,10,13}$ Unfortunately, among patients who don't undergo surgery, half die within 1 year of diagnosis. ${ }^{13}$

For patients with locally unresectable H-CCA and those precluded from resection due to underlying liver disease (e.g. PSC), liver transplantation has become a promising option. Although the initial results with transplantation for H-CCA were dismal, efforts championed by the Mayo Clinic to establish a comprehensive protocol, which includes better patient selection combined with intensive preoperative chemoradiation, have led to dramatically improved survival. ${ }^{17-20}$ In 2000, De Veerde and colleagues from the Mayo clinic published their seminal series of 25 patients with H-CCA who were evaluated for transplantation. Nineteen patients were deemed transplant candidates, of whom 12 (63\%) ultimately underwent transplant. Importantly, of those who received a transplant, graft survival was $91 \%$ and no patients died after a median follow-up of 44 months. ${ }^{19}$

In 2005, Rea and colleagues reported an update to the initial Mayo series, and compared patients with unresectable H-CCA or underlying PSC who were treated with neoadjuvant chemoradiation followed by transplantation, as per their protocol, to patients who underwent resection for resectable disease. Of those enrolled in the transplant protocol, 54\% ( $\mathrm{n}=38)$ underwent liver transplantation; of those who underwent attempted resection, $48 \%(\mathrm{n}=26)$ were with curative-intent. The authors found improved 1-, 3-, and 5-year survivals among patients who underwent transplant $(92 \%, 82 \%$, and $82 \%$, respectively) compared to those who underwent curative-intent resection $(82 \%, 48 \%$, and $21 \%$, respectively), which persisted even among patients without PSC. However, in an intention-to-treat analysis, there was no difference in survival between groups. Still, the authors concluded that neoadjuvant therapy followed by transplantation should be considered as an alternative to resection among patients with node-negative, localized H-CCA. ${ }^{24}$

More recently, Croome et al. published a study comparing patients treated at the Mayo Clinic who underwent resection ( $\mathrm{n}=99)$ to those who underwent neoadjuvant therapy/ transplant (n=54) specifically for de novo H-CCA. Again, the authors found improved 1-, 3-, and 5-year survivals with transplantation (90\%, $71 \%$, and 59\%, respectively) compared to resection $(81 \%, 53 \%$, and $36 \%$, respectively). Intention-to-treat analysis similarly demonstrated improved survival with transplantation over resection. However, on subgroup analysis of patients with $\mathrm{R} 0$ resection and $\mathrm{N} 0$ disease, no difference between groups was seen. Thus, the authors concluded that patients with clearly resectable de novo H-CCA should undergo resection. ${ }^{25}$

In the current study of 304 patients with H-CCA, 234 underwent attempted resection, while 70 patients were enrolled in a transplant protocol. Curative-intent resection was achieved in $82 \%$ of those explored, which is higher than what is reported in the literature, and may reflect better preoperative imaging techniques or variations in patient selection and definitions of respectability in a more modern cohort. ${ }^{7,13}$ Still, the margin positive, morbidity, and perioperative mortality rates following resection were similar to what has been previously reported.$^{5-9,13}$ Of those enrolled in a transplant protocol, $66 \%$ ultimately 
underwent transplantation, which is in line with the reported experience at Mayo, as well as other centers across the country. ${ }^{21,24}$

In order to better match patient groups in our study, an additional 5 patients who completed the neoadjuvant therapy protocol and underwent transplantation were excluded due to unconfirmed diagnoses of H-CCA. Despite strict clinical diagnostic criteria, a pathologic diagnosis in patients with suspected H-CCA can be challenging. Benign strictures, particularly in patients with PSC, often mimic malignancy, and it can be difficult to differentiate the two based on labs and imaging. ${ }^{26,27}$ Unfortunately, intraluminal biopsy and brushings are positive only approximately $30 \%$ of the time, and transabdominal biopsy automatically excludes patients from transplant consideration due to concerns for tumor seeding. ${ }^{19,21,28}$ Even using fluorescent in-situ hybridization, only $50 \%$ are positive. ${ }^{21}$ Furthermore, Lehrke et al. reported that $52 \%$ of patients who underwent transplant for clinically diagnosed H-CCA had no evidence of disease on final pathology. ${ }^{28}$ How many of those were due to true a pathologic complete response versus being merely a benign stricture is unclear. In a study by Darwish Murad et al. of 287 transplant patients with clinically diagnosed H-CCA from 12 U.S. centers, despite $75 \%$ successful transplantation, $30 \%$ of patients included had a no preoperative tissue diagnosis, at least an additional $14 \%$ had a suspected, but not confirmed, preoperative tissue diagnosis, and 54\% of patients had no tissue diagnosis on explant. ${ }^{21}$

Even after excluding those patients in our study without a pathologic diagnosis of H-CCA, still successful transplantation was achieved in 59\%. There were several obvious clinicopathologic differences between the transplant and resection groups. Transplant patients were younger, had slightly higher INR, and were more likely to have PSC, lymph node negative disease, and undergo neoadjuvant therapy compared to resection patients. The distribution of AJCC T-stage and Bismuth-Corlette classifications were also different between groups. However, all of these factors were used to select patients specifically for one surgical approach over the other, and the differences are to be expected. Higher ASA class and fewer R1 resections among transplant patients were also not unexpected.

Among all patients who underwent curative-intent surgery for pathologically-diagnosed $\mathrm{H}$ CCA, transplantation was associated with improved survival over resection (5-yr OS: 64\% vs $18 \%)$. Even when selecting out only $<3 \mathrm{~cm} / \mathrm{N} 0$ resection patients, which mimics the selection criteria for patients being considered for transplantation, 5-year OS after resection was only $31 \%$. However, these comparisons do include patients with PSC, who are typically not eligible for resection, and for whom transplantation is the standard-of-care. H-CCA in the background of PSC is also associated with improved survival compared to de novo disease. Thus, in order to focus on just the patients who might have otherwise been considered for either approach, outside of resectability, and to remove the associated survival bias, all those with underlying PSC were excluded. Still, transplantation was associated with improved survival over resection (5-yr OS: $54 \%$ vs $29 \%$ ). Even after further isolating only patients who underwent $\mathrm{R} 0$ resection, which is a factor that is unknown preoperatively and cannot be used as a selection criterion, but is well-recognized as a poor prognostic factor, improved survival with transplantation was still seen. It is important to note, however, that the pathologic characteristics that were used to select out patients undergoing resection 
(tumor size and nodal status) were based entirely on final pathologic analysis, while actual patient selection criteria for transplantation are primarily based on preoperative evaluation, which can be inaccurate. Indeed, among the non-PSC transplant patients in the current study, who presumably met the upfront diagnostic criteria, including tumors $<3 \mathrm{~cm}$ in size on imaging and no evidence of lymph node disease, $50 \%$ actually had tumors larger than $3 \mathrm{~cm}$ on pathologic examination, and 19\% had lymph node positive disease. Yet even with this bias, transplantation was associated with improved survival.

No comparison of neoadjuvant therapy/transplant versus resection for malignancy would be adequate without intention-to-treat analysis. This is particularly true for H-CCA, where resection is largely performed upfront, while transplantation is delayed until sufficient workup and an intensive neoadjuvant therapy regimen are completed. As previously mentioned, the proportion of patients who started a transplant neoadjuvant protocol but did not undergo transplantation due to disease progression or death was similar to what is reported in the literature. Compared to all patients who underwent attempted resection, patients who were enrolled in a transplant protocol had improved OS. More importantly, however, when all patients who entered a transplant protocol were compared to all $<3 \mathrm{~cm} / \mathrm{N} 0$ patients who underwent attempted resection, transplant patients still had better survival, even when excluding those with PSC (5-yr OS: $41 \%$ vs $27 \%$ ).

This study has several limitations. First, its retrospective design and small sample size limits our ability to draw definitive conclusions from the results. As previously mentioned, there was a clear selection bias between patients who received a transplant and those who underwent resection. Given the limited sample size, particularly within patient subsets, methods to overcome these biases, such as propensity matching or multivariable analyses, were not possible. It is also important to note that while the survival following transplantation in the current study is similar to what has been previously been reported, survival following resection in the current study is lower than what is reported in some studies. However, survival following resection for H-CCA varies widely in the literature, ranging from $10 \%$ to $47 \%$ at 5 years, even in more modern series. Finally, although very similar, the transplant protocols amongst the three institutions were not identical, nor were resections standardized. However, to our knowledge, this is the first U.S.-based multiinstitutional study to examine the influence of transplantation versus resection on OS for patients with H-CCA, in an intention-to-treat fashion. Furthermore, by performing subset analyses, we attempted to isolate the cohorts to match patients who would otherwise be eligible for either transplant or resection based on standardized inclusion and exclusion criteria.

\section{Conclusion}

In conclusion, resection for hilar cholangiocarcinoma that meets criteria for transplant $(<3 \mathrm{~cm}$ and lymph node negative disease) is associated with significantly decreased survival compared to neoadjuvant therapy/transplantation for the same criteria with unresectable disease, even when taking into account unconfirmed pathologic diagnosis of H-CCA and PSC, and when performing intention-to-treat analyses. How the results of this study should be optimally applied to the current management of H-CCA, however, is still unclear, and 
requires further investigation and thoughtful consideration beyond what is addressed in the current study. For example, the liver and biliary tract injury associated with the intensive neoadjuvant protocol required for transplant eligibility can be significant, and leaves the vast majority of patients with few other treatment options, should they ultimately not undergo transplantation. Furthermore, the organ allocation system in the U.S. is ever evolving, and new policies, such as "Share 35," which regionalizes the sharing of livers beyond each individual organ procurement organization for patients with MELD scores of $\geq 35$, may change how and when organs are allocated to patients with malignancy. Although no significant changes to wait-times for patients listed for hepatocellular carcinoma have been demonstrated across all regions, how "Share 35" will specifically affect patients with HCCA remains to be seen. It is important to note that the majority of the transplants included in this study were performed prior to "Share 35" taking effect. Whether the results and outcomes of studies like this should guide and inform the timing and granting of MELD exception points for patients with H-CCA within "Share 35 " is not known. ${ }^{29}$ Given the improved survival with transplantation in an intention-to-treat analysis, however, it does suggest that a more generous policy of granting MELD exception points is warranted than currently allocated. Still, prospective studies and trials are needed in order to define the optimal treatment for this rare disease.

\section{Acknowledgments}

Sources of Support: None

\section{References}

1. Nakeeb A, Pitt HA, Sohn TA, et al. Cholangiocarcinoma. A spectrum of intrahepatic, perihilar, and distal tumors. Annals of surgery. 1996; 224(4):463-473. discussion 473-465. [PubMed: 8857851]

2. Mansour JC, Aloia TA, Crane CH, Heimbach JK, Nagino M, Vauthey JN. Hilar cholangiocarcinoma: expert consensus statement. HPB (Oxford). 2015; 17(8):691-699. [PubMed: 26172136]

3. Burak K, Angulo P, Pasha TM, Egan K, Petz J, Lindor KD. Incidence and risk factors for cholangiocarcinoma in primary sclerosing cholangitis. The American journal of gastroenterology. 2004; 99(3):523-526. [PubMed: 15056096]

4. Suarez-Munoz MA, Fernandez-Aguilar JL, Sanchez-Perez B, et al. Risk factors and classifications of hilar cholangiocarcinoma. World journal of gastrointestinal oncology. 2013; 5(7):132-138. [PubMed: 23919107]

5. Miyazaki M, Kimura F, Shimizu H, et al. One hundred seven consecutive surgical resections for hilar cholangiocarcinoma of Bismuth types II, III, IV between 2001 and 2008. J Hepatobiliary Pancreat Sci. 2010; 17(4):470-475. [PubMed: 19936600]

6. van Gulik TM, Ruys AT, Busch OR, Rauws EA, Gouma DJ. Extent of liver resection for hilar cholangiocarcinoma (Klatskin tumor): how much is enough? Digestive surgery. 2011; 28(2):141147. [PubMed: 21540600]

7. Jarnagin WR, Fong Y, DeMatteo RP, et al. Staging, resectability, and outcome in 225 patients with hilar cholangiocarcinoma. Annals of surgery. 2001; 234(4):507-517. discussion 517-509. [PubMed: 11573044]

8. Nuzzo G, Giuliante F, Ardito F, et al. Improvement in perioperative and long-term outcome after surgical treatment of hilar cholangiocarcinoma: results of an Italian multicenter analysis of 440 patients. Archives of surgery. 2012; 147(1):26-34. [PubMed: 22250108] 
9. Nagino M, Ebata T, Yokoyama Y, et al. Evolution of surgical treatment for perihilar cholangiocarcinoma: a single-center 34 -year review of 574 consecutive resections. Annals of surgery. 2013; 258(1):129-140. [PubMed: 23059502]

10. Cho MS, Kim SH, Park SW, et al. Surgical outcomes and predicting factors of curative resection in patients with hilar cholangiocarcinoma: 10-year single-institution experience. Journal of gastrointestinal surgery : official journal of the Society for Surgery of the Alimentary Tract. 2012; 16(9):1672-1679. [PubMed: 22798185]

11. Shimizu H, Kimura F, Yoshidome H, et al. Aggressive surgical resection for hilar cholangiocarcinoma of the left-side predominance: radicality and safety of left-sided hepatectomy. Annals of surgery. 2010; 251(2):281-286. [PubMed: 20054275]

12. Groot Koerkamp B, Wiggers JK, Allen PJ, et al. Recurrence Rate and Pattern of Perihilar Cholangiocarcinoma after Curative Intent Resection. Journal of the American College of Surgeons. 2015; 221(6):1041-1049. [PubMed: 26454735]

13. Matsuo K, Rocha FG, Ito K, et al. The Blumgart preoperative staging system for hilar cholangiocarcinoma: analysis of resectability and outcomes in 380 patients. Journal of the American College of Surgeons. 2012; 215(3):343-355. [PubMed: 22749003]

14. Lee SG, Song GW, Hwang S, et al. Surgical treatment of hilar cholangiocarcinoma in the new era: the Asan experience. J Hepatobiliary Pancreat Sci. 2010; 17(4):476-489. [PubMed: 19851704]

15. Rocha FG, Matsuo K, Blumgart LH, Jarnagin WR. Hilar cholangiocarcinoma: the Memorial Sloan-Kettering Cancer Center experience. J Hepatobiliary Pancreat Sci. 2010; 17(4):490-496. [PubMed: 19806295]

16. Valle J, Wasan H, Palmer DH, et al. Cisplatin plus gemcitabine versus gemcitabine for biliary tract cancer. The New England journal of medicine. 2010; 362(14):1273-1281. [PubMed: 20375404]

17. Meyer CG, Penn I, James L. Liver transplantation for cholangiocarcinoma: results in 207 patients. Transplantation. 2000; 69(8):1633-1637. [PubMed: 10836374]

18. Robles R, Figueras J, Turrion VS, et al. Spanish experience in liver transplantation for hilar and peripheral cholangiocarcinoma. Annals of surgery. 2004; 239(2):265-271. [PubMed: 14745336]

19. De Vreede I, Steers JL, Burch PA, et al. Prolonged disease-free survival after orthotopic liver transplantation plus adjuvant chemoirradiation for cholangiocarcinoma. Liver Transpl. 2000; 6(3): 309-316. [PubMed: 10827231]

20. Heimbach JK, Gores GJ, Haddock MG, et al. Liver transplantation for unresectable perihilar cholangiocarcinoma. Seminars in liver disease. 2004; 24(2):201-207. [PubMed: 15192792]

21. Darwish Murad S, Kim WR, Harnois DM, et al. Efficacy of neoadjuvant chemoradiation, followed by liver transplantation, for perihilar cholangiocarcinoma at 12 US centers. Gastroenterology. 2012; 143(1):88-98 e83. quiz e14. [PubMed: 22504095]

22. Gores GJ, Gish RG, Sudan D, Rosen CB, Group MES. Model for end-stage liver disease (MELD) exception for cholangiocarcinoma or biliary dysplasia. Liver Transpl. 2006; 12 (12 Suppl 3):S9597. [PubMed: 17123289]

23. Edge, SB.Byrd, DR.Compton, CC.Fritz, AG.Greene, FL., T, A., III, editors. AJCC Cancer Staging Manual. 7th. New York: Springer; 2010. Intrahepatic Bile Ducts; p. 201-209.

24. Rea DJ, Heimbach JK, Rosen CB, et al. Liver transplantation with neoadjuvant chemoradiation is more effective than resection for hilar cholangiocarcinoma. Annals of surgery. 2005; 242(3):451458. discussion 458-461. [PubMed: 16135931]

25. Croome KP, Rosen CB, Heimbach JK, Nagorney DM. Is Liver Transplantation Appropriate for Patients with Potentially Resectable De Novo Hilar Cholangiocarcinoma? Journal of the American College of Surgeons. 2015; 221(1):130-139. [PubMed: 25872685]

26. Corvera CU, Blumgart LH, Darvishian F, et al. Clinical and pathologic features of proximal biliary strictures masquerading as hilar cholangiocarcinoma. Journal of the American College of Surgeons. 2005; 201(6):862-869. [PubMed: 16310689]

27. Koea J, Holden A, Chau K, McCall J. Differential diagnosis of stenosing lesions at the hepatic hilus. World journal of surgery. 2004; 28(5):466-470. [PubMed: 15085401]

28. Lehrke HD, Heimbach JK, Wu TT, et al. Prognostic Significance of the Histologic Response of Perihilar Cholangiocarcinoma to Preoperative Neoadjuvant Chemoradiation in Liver Explants. The American journal of surgical pathology. 2016; 40(4):510-518. [PubMed: 26752544] 
29. Croome KP, Lee DD, Harnois D, Taner CB. Effects of the Share 35 Rule on Waitlist and Liver Transplantation Outcomes for Patients with Hepatocellular Carcinoma. PloS one. 2017; 12(1):e0170673. [PubMed: 28122003] 


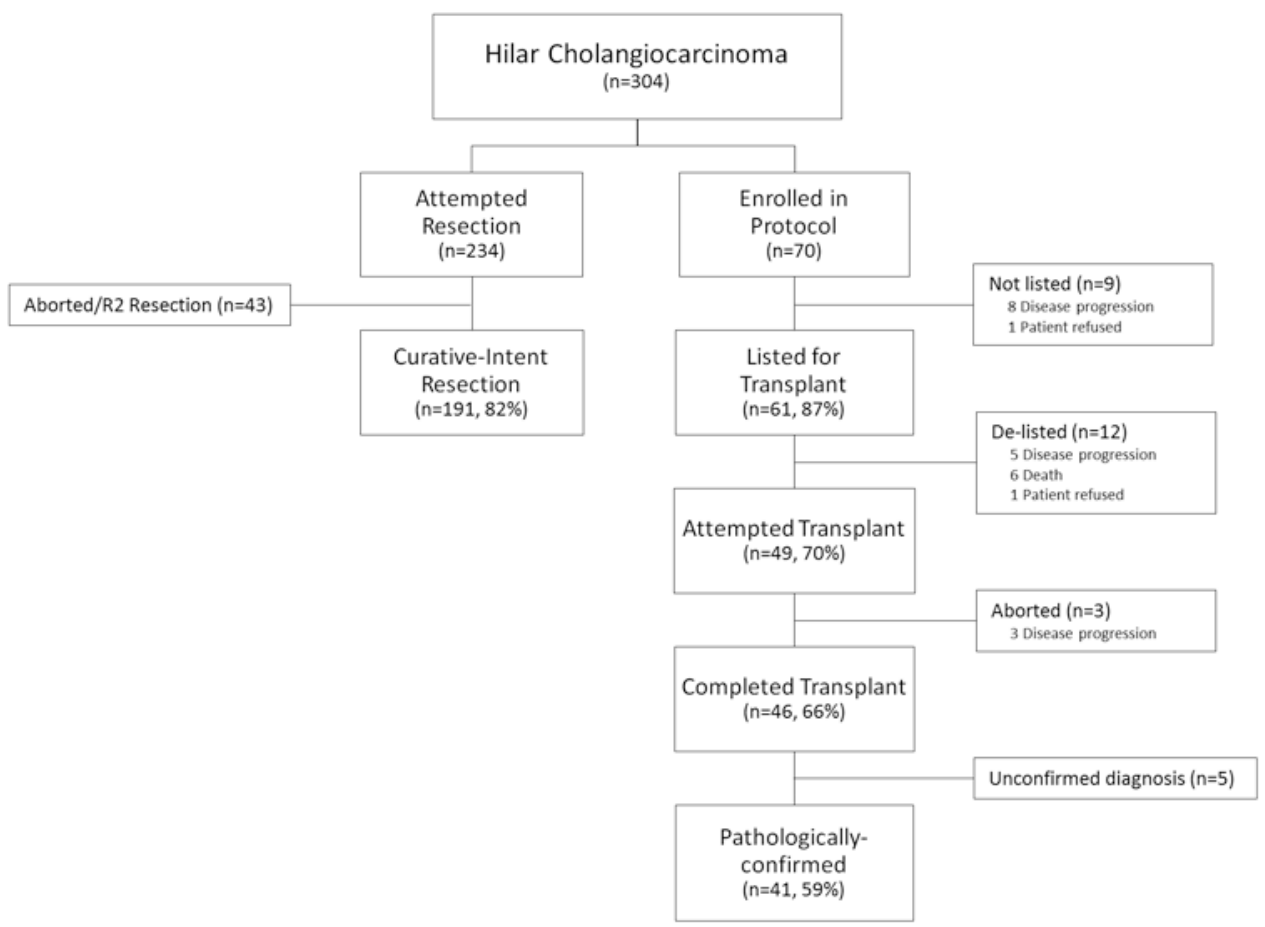

Figure 1.

Flow diagram of patients with hilar cholangiocarcinoma included in the current study. 

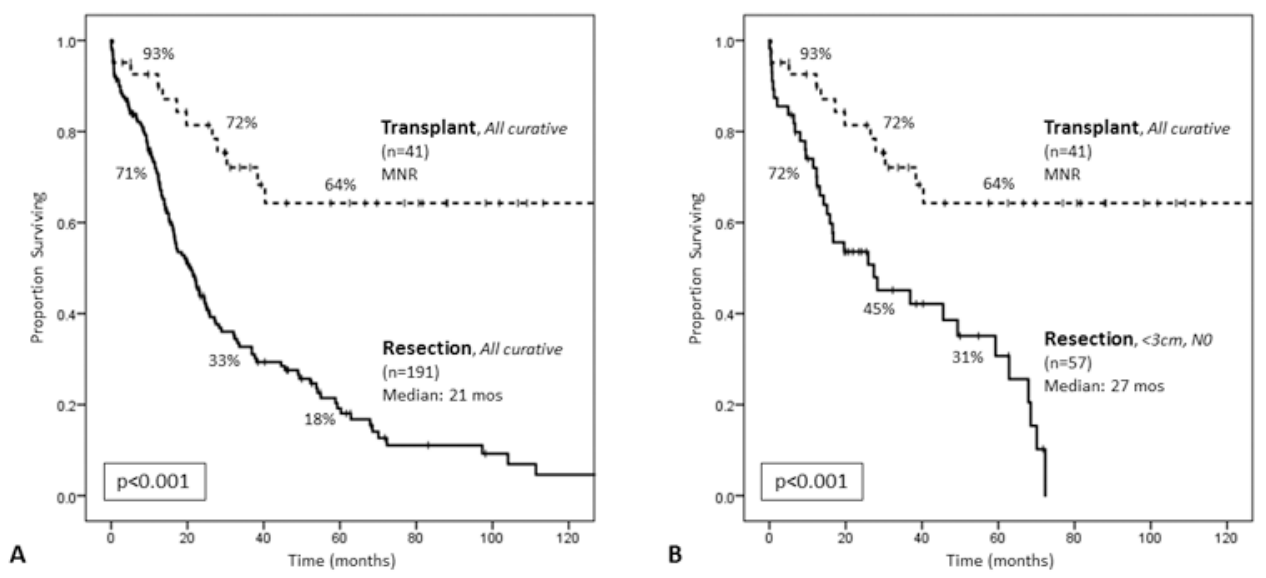

Figure 2.

(A) Comparing all patients with pathologically-confirmed H-CCA who underwent curativeintent transplant $(n=41)$ to those who underwent curative-intent resection $(n=191)$. The median survival was not reached (MNR) among transplant patients, and 1-, 3-, and 5-year survivals were $93 \%, 72 \%$, and $64 \%$, respectively. The median survival among resection patients was 21.0 months (95\% CI, 16.5-25.5), and 1-, 3-, and 5-year survivals were 71\%, $33 \%$, and $18 \%$, respectively (log rank $\mathrm{p}<0.001)$. (B) Comparing all patients with pathologically-confirmed H-CCA who underwent curative-intent transplant to those who underwent curative-intent resection and had tumors $<3 \mathrm{~cm}$ in size and no lymph node disease (N0) on pathologic examination $(n=57)$. The median survival for this subset of resection patients was 27.4 months (95\% CI, 6.0-48.8), and 1-, 3-, and 5-year survivals were 72\%, $45 \%$, and $31 \%$, respectively (log rank $\mathrm{p}<0.001)$. 

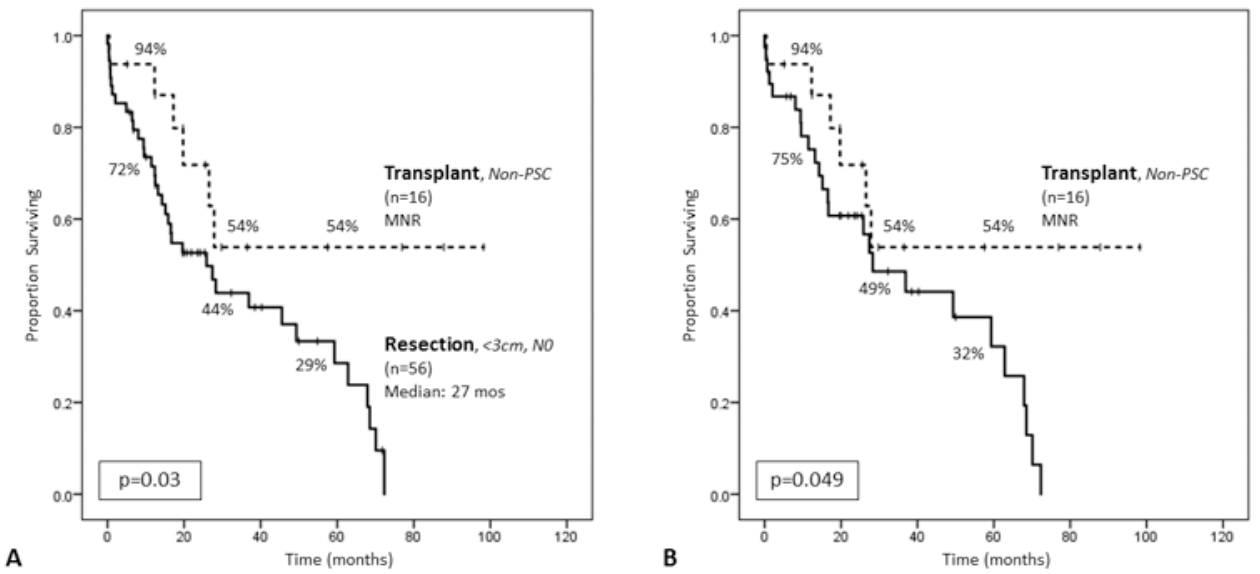

Figure 3.

(A) Comparing curative-intent transplantation for pathologically-confirmed H-CCA in nonPSC patients $(n=16)$ to curative-intent resection in non-PSC patients with tumors $<3 \mathrm{~cm}$ and no lymph node disease (N0) on pathologic examination $(n=56)$. The median survival was not reached (MNR) among non-PSC transplant patients, and 1-, 3-, and 5-year survivals were $94 \%, 54 \%$, and $54 \%$, respectively. The median survival for $<3 \mathrm{~cm} / \mathrm{N} 0$ resection patients was 25.9 months (95\% CI 13.0-38.7), and 1-, 3-, and 5-year survivals were 72\%, 44\%, and 29\%, respectively ( $\log$ rank $\mathrm{p}=0.03)$. $(\mathbf{B})$ Comparing pathologically-confirmed, non-PSC transplant patients to non-PSC, $<3 \mathrm{~cm} / \mathrm{N} 0$ resection patients who also had $\mathrm{R} 0$ resections $(\mathrm{n}=39)$. The median survival for this subset of resection patients was 28.3 months (95\% CI 12.6-44.0), and 1-, 3-, and 5-year survivals were $75 \%, 49 \%$, and 32\%, respectively (log rank p=0.049). 

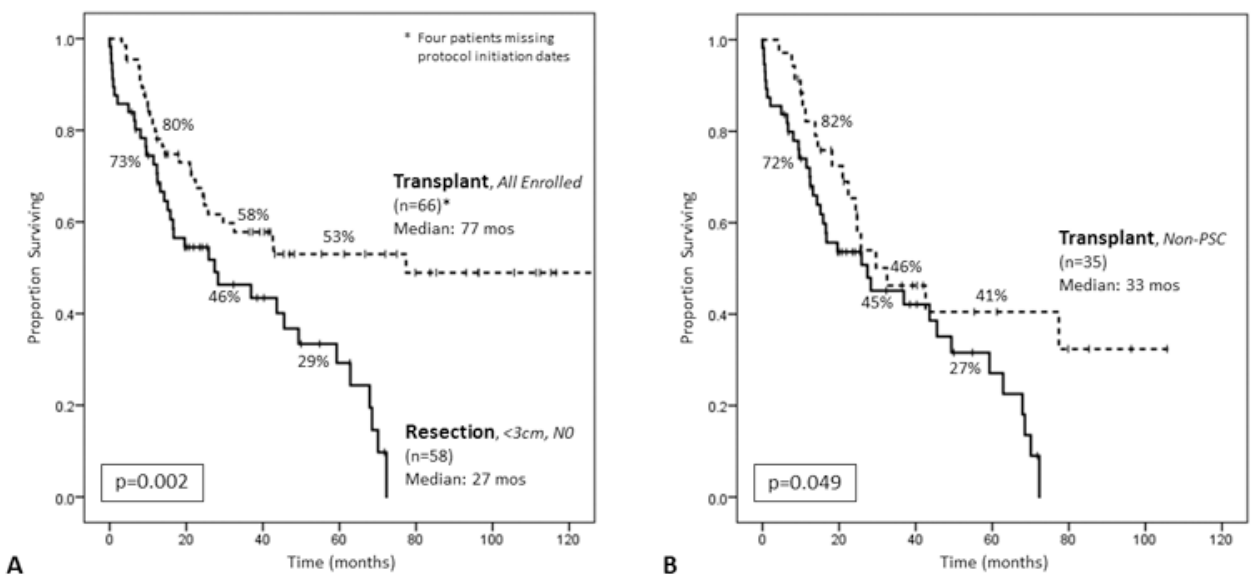

Figure 4.

Intention-to-treat analysis. (A)Comparing all patients enrolled in a transplant protocol $(\mathrm{n}=66)$ to all patients who underwent attempted resection with tumors $<3 \mathrm{~cm}$ and no lymph node disease (N0) on pathologic examination $(n=58)$. Median overall survival among transplant patients was 77.4 months, and 1-, 3-, and 5-year survivals were $80 \%, 58 \%$, and $53 \%$, respectively. Median overall survival among resection patients was 27.4 months (95\% CI, 5.5-49.3), and 1-, 3-, and 5-year survivals were 73\%, 46\%, 29\%, respectively (log rank $\mathrm{p}=0.002)$. (B) Comparing all non-PSC patients enrolled in a transplant protocol $(\mathrm{n}=35)$ to all patients who underwent attempted resection with tumors $<3 \mathrm{~cm}$ and $\mathrm{N} 0$ disease on pathologic examination $(\mathrm{n}=57)$. Median overall survival among transplant patients was 32.5 months (95\% CI, 13.5-51.5), and 1-, 3-, and 5-year survivals were 82\%, 46\%, and 41\%, respectively. Median overall survival among resection patients was 27.4 months $(95 \% \mathrm{CI}$, 6.0-48.8), and 1-, 3-, and 5-year survivals were $72 \%, 45 \%$, and $27 \%$, respectively (log rank $\mathrm{p}=0.049)$. 
Table 1

Comparison of clinicopathologic factors between patients who underwent curative-intent resection and curative-intent transplatation

\begin{tabular}{|c|c|c|c|}
\hline Variable & Resection $(\mathrm{n}=191,82 \%)$ & Transplant $(n=41,18 \%)$ & p-value \\
\hline Age (yrs), median (IQR) & $67(57-73)$ & $54(43-62)$ & $<0.001$ \\
\hline Male, n (\%) & $112(59)$ & $29(71)$ & 0.15 \\
\hline BMI (kg/m2), median (IQR) & $25(22-29)$ & $25(22-28)$ & 0.52 \\
\hline Race, n (\%) & & & 0.41 \\
\hline White & $148(79)$ & $36(88)$ & \\
\hline African-American & $14(7)$ & $2(5)$ & \\
\hline Other & $25(14)$ & $3(7)$ & \\
\hline ASA Class, n (\%) & & & $<0.001$ \\
\hline $1 / 2$ & $49(31)$ & $2(5)$ & \\
\hline $3 / 4$ & $109(69)$ & $37(95)$ & \\
\hline \multicolumn{4}{|l|}{ Preoperative Labs, median (IQR) } \\
\hline Bilirubin (mg/dL) & $1.7(0.9-3.6)$ & $0.8(0.5-2.3)$ & 0.001 \\
\hline Albumin $(\mathrm{g} / \mathrm{dL})$ & $3.4(2.8-3.8)$ & $3.6(2.9-3.8)$ & 0.32 \\
\hline Creatinine $(\mathrm{mg} / \mathrm{dL})$ & $0.8(0.7-1.0)$ & $0.8(0.7-1.0)$ & 0.97 \\
\hline INR & $1.0(1.0-1.1)$ & $1.1(1.0-1.2)$ & 0.004 \\
\hline Primary Sclerosing Cholangitis, n (\%) & $3(2)$ & $25(61)$ & $<0.001$ \\
\hline \multicolumn{4}{|l|}{ Type of Resection, $\mathrm{n}(\%)$} \\
\hline Hemi-Hepatectomy + CBD Resection & $90(47)$ & & \\
\hline Extended Hepatectomy + CBD Resection & $58(30)$ & - & - \\
\hline Trisectionectomy + CBD Resection & $43(22)$ & & \\
\hline Major Complication, n (\%) & $82(45)$ & $14(34)$ & 0.30 \\
\hline Postoperative Liver Failure & $12(7)$ & $4(10)$ & 0.50 \\
\hline 30-day Mortality & $15(8)$ & $2(5)$ & 0.74 \\
\hline 90-day Mortality & $22(12)$ & $2(5)$ & 0.27 \\
\hline Length of Stay (days), median (IQR) & $11(7-18)$ & $8(6-14)$ & 0.03 \\
\hline Pathologic complete response, n (\%) & - & $12(29)$ & - \\
\hline Final margin status, $\mathrm{n}(\%)$ & & & 0.01 \\
\hline R0 & $134(70)$ & $36(90)$ & \\
\hline R1 & $57(30)$ & $4(10)$ & \\
\hline Tumor size, median (IQR) & $3.0(2.0-4.0)$ & $2.5(1.1-5.0)$ & 0.51 \\
\hline AJCC T-Stage, n (\%) & & & 0.002 \\
\hline $\mathrm{Tis} / \mathrm{T} 1$ & $15(10)$ & $8(29)$ & \\
\hline
\end{tabular}




\begin{tabular}{|c|c|c|c|}
\hline Variable & Resection $(n=191,82 \%)$ & Transplant $(n=41,18 \%)$ & p-value \\
\hline $\mathrm{T} 2$ & $100(68)$ & $12(44)$ & \\
\hline $\mathrm{T} 3$ & $28(19)$ & $5(19)$ & \\
\hline $\mathrm{T} 4$ & $4(3)$ & $2(7)$ & \\
\hline Blumgart T-Stage, n (\%) & & & 0.12 \\
\hline $\mathrm{T} 1$ & $81(46)$ & $15(68)$ & \\
\hline $\mathrm{T} 2$ & $42(34)$ & $2(9)$ & \\
\hline $\mathrm{T} 3$ & $53(30)$ & $5(23)$ & \\
\hline Bismuth Classification, n (\%) & & & $<0.001$ \\
\hline Type I & $8(5)$ & $0(0)$ & \\
\hline Type II & $23(13)$ & $16(70)$ & \\
\hline Type III & $108(61)$ & $2(9)$ & \\
\hline Type IV & $37(21)$ & $5(23)$ & \\
\hline Grade, n (\%) & & & 0.14 \\
\hline Low & $36(20)$ & $5(21)$ & \\
\hline Intermediate & $102(58)$ & $9(38)$ & \\
\hline High & $38(22)$ & $10(42)$ & \\
\hline Lymphovascular invasion, n (\%) & $63(41)$ & $7(18)$ & 0.02 \\
\hline Perineural invasion, $\mathrm{n}(\%)$ & $127(78)$ & $13(33)$ & $<0.001$ \\
\hline Lymph nodes retrieved, n (\%) & $169(91)$ & $33(83)$ & 0.17 \\
\hline \# Lymph nodes retrieved, median (IQR) & $4(2-7)$ & $3(1-5)$ & 0.04 \\
\hline Lymph node positive, n (\%) & $66(38)$ & $7(19)$ & 0.04 \\
\hline \multicolumn{4}{|l|}{ Neoadjuvant therapy, n (\%) } \\
\hline Chemotherapy & $9(5)$ & $39(95)$ & $<0.001$ \\
\hline Radiation & $6(3)$ & $39(95)$ & $<0.001$ \\
\hline \multicolumn{4}{|l|}{ Adjuvant therapy, n (\%) } \\
\hline Chemotherapy & $89(52)$ & $1(3)$ & $<0.001$ \\
\hline Radiation & $58(35)$ & $1(3)$ & $<0.001$ \\
\hline Recurrence, n (\%) & $65(37)$ & $10(24)$ & 0.19 \\
\hline Isolated locoregional & $18(29)$ & $2(20)$ & 0.72 \\
\hline Distant (liver, peritoneum, lung, other) & $45(71)$ & $8(80)$ & 0.72 \\
\hline Isolated locoregional and/or liver only & $39(62)$ & $2(20)$ & 0.02 \\
\hline
\end{tabular}

BMI, mass body index; IQR, interquartile range; ASA, American Society of Anesthesiologists; INR, international normalized ratio; CBD, common bile duct; AJCC, American Joint Committee on Cancer 
Table 2

Comparison of clinicopathologic factors between patients who underwent curative-intent resection $(<3 \mathrm{~cm}, \mathrm{NO})$ and curative-intent transplantation, excluding patients primary sclerosing cholangitis

\begin{tabular}{|c|c|c|c|}
\hline Variable & $\begin{array}{c}\text { Resection } \\
\text { Curative, }<3 \mathrm{~cm}, \mathrm{NO} \\
(\mathrm{n}=56,78 \%)\end{array}$ & $\begin{array}{c}\text { Transplant } \\
\text { Non-PSC } \\
(\mathrm{n}=16,22 \%)\end{array}$ & p-value \\
\hline Age (yrs), median (IQR) & $67(58-74)$ & $60(50-66)$ & 0.01 \\
\hline Male, n (\%) & $32(57)$ & $11(69)$ & 0.56 \\
\hline BMI $(\mathrm{kg} / \mathrm{m} 2)$, median (IQR) & $26(23-29)$ & $25(23-28)$ & 0.51 \\
\hline Race, n (\%) & & & 0.29 \\
\hline White & $41(73)$ & $13(81)$ & \\
\hline African-American & $5(9)$ & $2(13)$ & \\
\hline Other & $10(18)$ & $1(6)$ & \\
\hline ASA Class, n (\%) & & & $<0.001$ \\
\hline $1 / 2$ & $15(33)$ & $1(6)$ & \\
\hline $3 / 4$ & $30(67)$ & $15(94)$ & \\
\hline \multicolumn{4}{|c|}{ Preoperative Labs, median (IQR) } \\
\hline Bilirubin (mg/dL) & $2.0(0.9-4.2)$ & $0.7(0.4-1.6)$ & 0.008 \\
\hline Albumin (g/dL) & $3.4(3.1-3.8)$ & $3.6(3.0-3.8)$ & 0.67 \\
\hline Creatinine $(\mathrm{mg} / \mathrm{dL})$ & $0.8(0.7-0.9)$ & $0.7(0.6-0.9)$ & 0.21 \\
\hline INR & $1.0(1.0-1.1)$ & $1.1(1.0-1.2)$ & 0.031 \\
\hline
\end{tabular}

Type of Resection, n (\%)

\begin{tabular}{lccc} 
Hemi-Hepatectomy + CBD Resection & $32(58)$ & & - \\
Extended Hepatectomy + CBD Resection & $17(30)$ & & \\
Trisectionectomy + CBD Resection & $7(12)$ & & \\
\hline Major Complication, n (\%) & $22(42)$ & $4(25)$ & 0.38 \\
Postoperative Liver Failure & $4(7)$ & $2(23)$ & 0.61 \\
30-day Mortality & $6(11)$ & $1(6)$ & 1.00 \\
90-day Mortality & $8(14)$ & $1(6)$ & 0.67 \\
\hline Length of Stay (days), median (IQR) & $9(7-17)$ & $7(5-13)$ & 0.17 \\
\hline Pathologic complete response, n (\%) & - & $0(0)$ & - \\
\hline Final margin status, n (\%) & $39(70)$ & $13(81)$ & 0.01 \\
R0 & $17(30)$ & $3(19)$ & \\
\hline R1 & $2.0(1.5-2.5)$ & $2.9(1.2-5.5)$ & 0.08 \\
\hline Tumor size, median (IQR) & & & 0.09
\end{tabular}

AJCC T-Stage, n (\%)

0.09 


\begin{tabular}{|c|c|c|c|}
\hline Variable & $\begin{array}{c}\text { Resection } \\
\text { Curative, }<3 \mathrm{~cm}, N 0 \\
(\mathrm{n}=\mathbf{5 6}, \mathbf{7 8 \%})\end{array}$ & $\begin{array}{c}\text { Transplant } \\
\text { Non-PSC } \\
(\mathrm{n}=16,22 \%)\end{array}$ & p-value \\
\hline Tis/T1 & $9(24)$ & $5(31)$ & \\
\hline $\mathrm{T} 2$ & $24(62)$ & $6(38)$ & \\
\hline $\mathrm{T} 3$ & $5(13)$ & $3(19)$ & \\
\hline $\mathrm{T} 4$ & $0(0)$ & $2(13)$ & \\
\hline Blumgart T-Stage, $\mathrm{n}(\%)$ & & & 0.19 \\
\hline $\mathrm{T} 1$ & $20(40)$ & $9(60)$ & \\
\hline $\mathrm{T} 2$ & $14(28)$ & $1(7)$ & \\
\hline $\mathrm{T} 3$ & $16(32)$ & $5(33)$ & \\
\hline Bismuth Classification, $\mathrm{n}(\%)$ & & & $<0.001$ \\
\hline Type I & $3(6)$ & $0(0)$ & \\
\hline Type II & $6(12)$ & $9(60)$ & \\
\hline Type III & $28(54)$ & $1(7)$ & \\
\hline Type IV & $15(29)$ & $5(33)$ & \\
\hline Grade, n $(\%)$ & & & 0.10 \\
\hline Low & $11(22)$ & $0(0)$ & \\
\hline Intermediate & $31(61)$ & $9(64)$ & \\
\hline High & $9(18)$ & $5(36)$ & \\
\hline Lymphovascular invasion, $\mathrm{n}(\%)$ & $14(30)$ & $5(31)$ & 1.00 \\
\hline Perineural invasion, $\mathrm{n}(\%)$ & $38(81)$ & $8(50)$ & 0.04 \\
\hline Lymph nodes retrieved, $\mathrm{n}(\%)$ & $56(100)$ & $14(88)$ & 0.05 \\
\hline \# Lymph nodes retrieved, median (IQR) & $3(2-6)$ & $4(2-6)$ & 0.85 \\
\hline Lymph node positive, n (\%) & $0(0)$ & $3(19)$ & 0.009 \\
\hline \multicolumn{4}{|l|}{ Neoadjuvant therapy, n (\%) } \\
\hline Chemotherapy & $3(5)$ & $16(100)$ & $<0.001$ \\
\hline Radiation & $2(4)$ & $16(100)$ & $<0.001$ \\
\hline \multicolumn{4}{|l|}{ Adjuvant therapy, n (\%) } \\
\hline Chemotherapy & $18(36)$ & $0(0)$ & 0.003 \\
\hline Radiation & $13(26)$ & $0(0)$ & 0.03 \\
\hline Recurrence, n (\%) & $15(29)$ & $5(31)$ & 1.00 \\
\hline Locoregional only & $4(31)$ & $1(20)$ & 1.00 \\
\hline Distant & $9(69)$ & $4(80)$ & \\
\hline
\end{tabular}

BMI, mass body index; IQR, interquartile range; ASA, American Society of Anesthesiologists; INR, international normalized ratio; CBD, common bile duct; AJCC, American Joint Committee on Cancer 